\title{
Complex spectrum of finite-density lattice QCD with static quarks at strong coupling
}

\author{
Hiromichi Nishimurd* \\ Institute for Theoretical Physics, Johann Wolfgang Goethe-University \\ Max-von-Laue-Str. 1, D-60438 Frankfurt am Main, Germany and \\ Department of Physics, Washington University, St. Louis, MO 63130 USA \\ Michael C. Ogilvie $\AA^{\dagger}$ and Kamal Pangen $\rceil^{\dagger}$ \\ Department of Physics, Washington University, St. Louis, MO 63130 USA
}

(Dated: $1 / 20 / 2016$ )

\begin{abstract}
We calculate the spectrum of transfer matrix eigenvalues associated with Polyakov loops in finitedensity lattice QCD with static quarks. These eigenvalues determine the spatial behavior of Polyakov loop correlations functions. Our results are valid for all values of the gauge coupling in $1+1$ dimensions, and valid in the strong-coupling region for any number of dimensions. When the quark chemical potential $\mu$ is nonzero, the spatial transfer matrix $T_{s}$ is non-Hermitian. The appearance of complex eigenvalues in $T_{s}$ is a manifestation of the sign problem in finite-density QCD. The invariance of finite-density QCD under the combined action of charge conjugation $\mathcal{C}$ and complex conjugation $\mathcal{K}$ implies that the eigenvalues of $T_{s}$ are either real or part of a complex pair. Calculation of the spectrum confirms the existence of complex pairs in much of the temperature-chemical potential plane. Many features of the spectrum for static quarks are determined by a particle-hole symmetry. For $\mu$ small compared to the quark mass $M$, we typically find real eigenvalues for the lowest lying states. At somewhat larger values of $\mu$, pairs of eigenvalues may form complex-conjugate pairs, leading to damped oscillatory behavior in Polyakov loop correlation functions. However, near $\mu=M$, the low-lying spectrum becomes real again. This is a direct consequence of the approximate particle-hole symmetry at $\mu=M$ for heavy quarks. This behavior of the eigenvalues should be observable in lattice simulations and can be used as a test of lattice algorithms. Our results provide independent confirmation of results we have previously obtained in PNJL models using complex saddle points.
\end{abstract}

\section{INTRODUCTION}

Although lattice simulations have given excellent first-principles results for many observables of finite-temperature $\mathrm{QCD}$, there has been less clear success when the chemical potential $\mu$ is nonzero. Finite-density QCD is one of the class of theoretical models that has a sign problem: the partition function is a sum over complex weights which cannot be interpreted as relative probabilities [1-3. Many methods have been used in attempts to overcome the sign problem in finite-density QCD. Two methods that have received significant attention recently are the complex Langevin technique [4 9] and the Lefschetz thimble approach [10 18. We have recently explored the implications of complex saddle points in phenomenological models of QCD at finite temperature and density [19, 20]. These models postulate effective potentials for the Polyakov loop $\operatorname{Tr}_{F} P_{x}$ and other order parameters in such a way that the confinement-deconfinement transition of quenched QCD is incorporated. The most realistic of these models are Polyakov-Nambu-Jona Lasinio (PNJL) models, and include the effects of chiral symmetry restoration [21]. In all the cases studied in [19, 20, a nonzero $\mu$ resulted in a complex saddle point for the eigenvalues of the Polyakov loop. A number of desirable results emerge from this. For example, the free energy is real at the complex saddle point and $\left\langle\operatorname{Tr}_{F} P\right\rangle \neq\left\langle\operatorname{Tr}_{F} P^{\dagger}\right\rangle$. The mass matrix for Polyakov loops exhibits a new feature: the mass eigenvalues may form a complex conjugate pair, indicating the occurrence of spatially-modulated sinusoidal decay. Such behavior is forbidden by spectral positivity for $\mu=0$, but is possible when $\mu \neq 0$. In the case of PNJL models, complex conjugate pairs occur in regions around the first-order line that emerges from $T=0$, terminating at a critical end-point.

Here we address the generality of this phenomenon by showing similar behavior in lattice QCD with static quarks in the strong-coupling limit. We will use a transfer matrix formalism to determine the behavior of Polyakov loop correlation functions as a function of spatial separation. These results are exact for any gauge coupling in $1+1$ dimensions, but also represent the leading-order result in the character expansion in higher dimensions. As the

\footnotetext{
*Electronic address: hnishimura@physics.wustl.edu

† Electronic address: mco@physics.wustl.edu

¥Electronic address: kamalpangeni@wustl.edu
} 
chemical potential of the static quarks is varied, we will show that there are large regions of parameter space where the eigenvalues of the transfer matrix form complex conjugate pairs, leading to damped oscillatory behavior of Polyakov loop correlation functions. The boundary of such a region in parameter space is referred to as a disorder line in condensed matter physics. The method used is completely different from the saddle point technique employed in [19, 20, applied to a very different model, illustrating the generality of the behavior. Any reliable simulation method for finite-density lattice QCD should be able to reproduce our results, which thus can serve as a benchmark for the validation of algorithms.

Section II describes the strong-coupling formalism underlying our calculation. We give a graphical demonstration of the non-hermiticity of the correlation function matrix in character space when the chemical potential is nonzero. We also discuss the symmetries of the model, paying particular attention to particle-hole symmetry. Section III explains how the transfer matrix for Polyakov loops can be realized in the character basis in a form suitable for numerical diagonalization. In section IV, we present our results for the Polyakov loop spectrum. A final section gives our conclusions.

\section{STRONG-COUPLING FORMALISM}

\section{A. Setup}

Strong-coupling expansions and character expansions are well-developed methods for exploring the properties of lattice gauge theories [22]. Strong-coupling expansions are typically expansions in inverse powers of some coupling $g^{2}$ around $1 / g^{2}=0$. Generally such expansions have a finite radius of convergence in an $1 / g^{2}$, and thus are not directly relevant for the continuum limit of non-Abelian gauge theories at $g^{2}=0$. Nevertheless, they have often given important insight into mechanisms and critical behavior. Character expansions are closely related to strong-coupling expansions, but have many advantages. Consider the case of $S U(3)$ lattice gauge fields in $1+1$ dimensions at some finite temperature. In the absence of non-gauge fields, i.e., the quenched approximation, this model is exactly solvable. The action $S_{p}$ of a single plaquette $U_{p}$ can be expanded in character expansion

$$
e^{S_{p}\left[U_{p}\right]}=\sum_{R} d_{R} c_{R} \chi_{R}\left(U_{p}\right)
$$

where $\chi_{R}$ is the character of an irreducible representation $R$ of the gauge group $G, d_{R}$ is the dimensionality of $R$, and $c_{R}$ is a coefficient that depends on the parameters of the gauge action $S_{p}$. The character expansion is an expansion in the ratios $c_{R} / c_{0}$, where $c_{0}$ is the coefficient of the trivial (identity) representation. A strong-coupling expansion in $1 / g^{2}$ may be obtained by expanding these ratios. We will be using the character expansion in what follows.

Our principle observable is the trace of the Polyakov loop operator $P_{x}$ in irreducible representations of $S U(3)$. The Polyakov loop operator $P_{x}$ is the time-ordered product of the timelike links starting at a given point $x$ and returning to that point due to the periodic boundary conditions of finite-temperature lattice gauge theories. The trace of $P_{x}$ in an irreducible representation $R$ measures confinement for that representation: $\left\langle\operatorname{Tr}_{R} P\right\rangle=\exp \left(-\beta F_{R}\right)$, where $F_{R}$ is the free energy required to insert a static particle in a representation $R$ into the system and $\beta$ is the inverse of the temperature $T$. In a pure gauge theory, the trace in the fundamental representation, $\operatorname{Tr}_{F} P$, is an order parameter for confinement.

We will begin by giving simple arguments that show that the Polyakov loop propagator matrix is not Hermitian at finite density, indicating the possibility of complex eigenvalues. The correlation function of Polyakov loops in a representation $R,\left\langle\operatorname{Tr}_{R} P_{x} \operatorname{Tr}_{R} P_{y}^{\dagger}\right\rangle$ is given to lowest order in the character expansion by tiling space with plaquettes in the representation $R$ between $x$ and $y$. We now introduce static quarks into this system. Each quark carries with it a factor of $\operatorname{Tr}_{F} P$ with an additional factor of $\exp (\beta \mu)$ when the chemical potential $\mu \neq 0$. As shown in Fig. 1 . this generates a new interaction not present in the quenched case that couples $\operatorname{Tr}_{F} P$ to itself. Correlation functions of the form $\left\langle\operatorname{Tr}_{R} P_{x} \operatorname{Tr}_{R} P_{y}\right\rangle$ have been measured in lattice simulation of full QCD at $\mu=0$ [23]. When $\mu \neq 0$, the lowest-order contribution to $\left\langle\operatorname{Tr}_{R} P_{x} \operatorname{Tr}_{R} P_{y}\right\rangle$ is enhanced by a weight factor $\exp (\beta \mu)$. On the other hand, the coupling of $\operatorname{Tr}_{F} P^{\dagger}$ to itself, as represented in Fig. 2, is suppressed by a corresponding factor of $\exp -\beta \mu$. The only difference between the two graphs is the factor of $\exp (\beta \mu)$ versus $\exp (-\beta \mu)$ so $\left\langle\operatorname{Tr}_{R} P_{x} \operatorname{Tr}_{R} P_{y}\right\rangle \neq\left\langle\operatorname{Tr}_{R} P_{x}^{\dagger} \operatorname{Tr}_{R} P_{y}^{\dagger}\right\rangle$. Thus when $\mu \neq 0$ the matrix of two-point correlation functions is no longer Hermitian. In order to find masses, that matrix of correlation functions must be diagonalized. However, if the correlation matrix is not Hermitian, the masses need not be real. The asymmetry between $\operatorname{Tr}_{F} P$ and $\operatorname{Tr}_{F} P^{\dagger}$ is a consequence of the explicit breaking of charge conjugation $\mathcal{C}$ by the chemical potential. However, the symmetry of finite-density QCD under the combined action of $\mathcal{C}$ and complex conjugation $\mathcal{K}$ remains intact [19, 20, 24, 25]. Thus the combined action of $\mathcal{C K}$ leaves $\operatorname{Tr}_{F} P$ and $\operatorname{Tr}_{F} P^{\dagger}$ invariant. For our purposes, the most important consequence of the $\mathcal{C K}$ symmetry is that the masses are either real or are part of a complex conjugate pair, as we discuss below. 


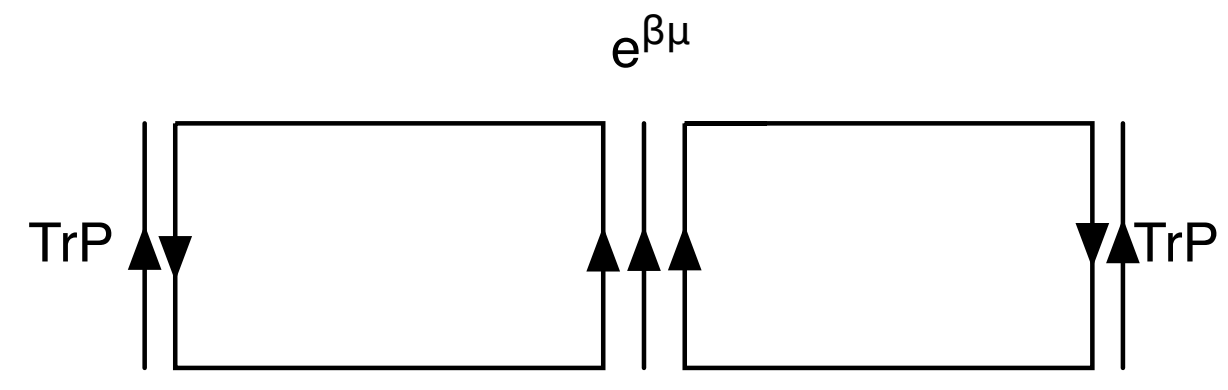

Figure 1: Graphical representation of a contribution of fermions to $\left\langle\operatorname{Tr}_{F} P \operatorname{Tr}_{F} P\right\rangle$

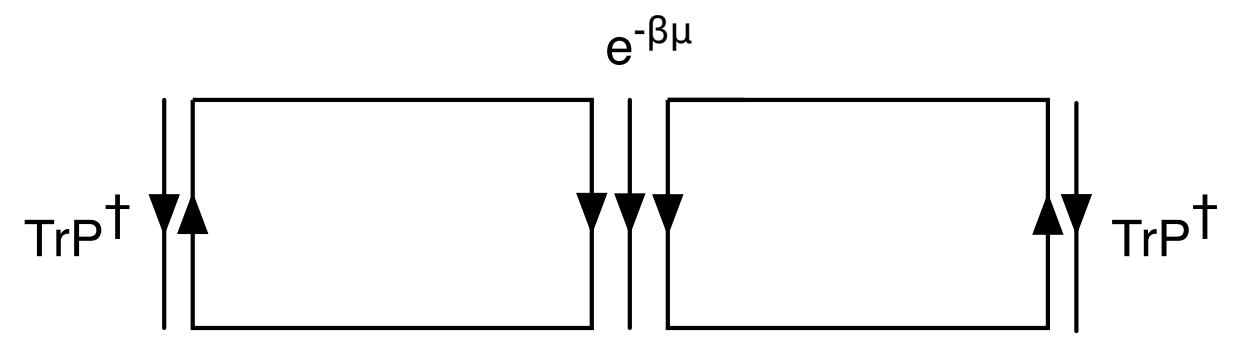

Figure 2: Graphical representation of a contribution of fermions to $\left\langle\operatorname{Tr}_{F} P^{\dagger} \operatorname{Tr}_{F} P^{\dagger}\right\rangle$.

A comprehensive treatment of Polyakov loop correlation functions at strong coupling is most conveniently carried out using a version of Svetitsky-Yaffe universality [26, 27]. This fundamental result for gauge theories at finite temperature links the critical behavior of Polyakov loops in pure gauge theories in $d$ dimensions with the behavior of spin systems in $d-1$ dimensions. Furthermore, the effect of introducing quarks into the gauge theory is similar to the effect of an external magnetic field on a spin system. It is easy in strong coupling to show that the effective action for the interaction of Polyakov loops is similar to that found in a spin system. If we consider two adjacent Polyakov loops on a lattice, they share a "belt" of plaquettes running up the time axis. Integrating over the spatial links of this belt leads to an effective interaction between the Polyakov loops of the form

$$
\sum_{R} c_{R}^{N_{t}} \chi_{R}\left(P_{x}\right) \chi_{R}\left(P_{y}^{\dagger}\right)
$$

where $P_{x}$ and $P_{y}$ are Polyakov loops on adjacent spatial lattice sites $x$ and $y$. The parameter $N_{t}$ is the temporal size of the lattice, so $\beta=N_{t} a$, where $a$ is the lattice spacing. To leading order in a strong-coupling expansion, the exponential of the effective action is

$$
e^{S_{e f f}}=\prod_{\langle x y\rangle}\left[\sum_{R} c_{R}^{N_{t}} \chi_{R}\left(P_{x}\right) \chi_{R}\left(P_{y}^{\dagger}\right)\right]
$$

where the product is taken over all nearest-neighbor spatial lattice sites. For very strong coupling, the contribution of the fundamental representation usually dominates, and the effective action may be written approximately as

$$
e^{S_{e f f}} \simeq \exp \left\{\sum_{\langle x y\rangle} J\left[\chi_{F}\left(P_{x}\right) \chi_{F}\left(P_{y}^{\dagger}\right)+\chi_{F}\left(P_{x}^{\dagger}\right) \chi_{F}\left(P_{y}\right)\right]\right\}
$$

where the sum over $\langle x y\rangle$ is a sum over nearest-neighbor pairs of spatial points and

$$
J=\left(\frac{c_{F}}{c_{0}}\right)^{N_{t}}
$$

This is clearly of the form of a spin system, with spins taking on values in $G$ and the interaction respecting global center symmetry. 
In order to arrive at a simple model, we consider only the case where quarks are so heavy that move only in time and are static in space. In this case, the effects of quarks at $x$ can be represented in the partition function by a weight 28

$$
D_{x}=\operatorname{det}\left[1+e^{\beta \mu-\beta M} P_{x}\right]
$$

while antiquarks give a weight factor

$$
\bar{D}_{x}=\operatorname{det}\left[1+e^{-\beta \mu-\beta M} P_{x}^{\dagger}\right]
$$

where $\mu$ is the chemical potential and $M$ is the heavy quark mass. It will be convenient to associate two different "activities" for quarks and antiquarks:

$$
\begin{aligned}
& z_{1}=e^{\beta \mu-\beta M} \\
& z_{2}=e^{-\beta \mu-\beta M}
\end{aligned}
$$

Although $z_{1}$ and $z_{2}$ may take on any non-negative values, their association with $\mu$ and $M$ does impose restrictions: Depending on the sign of $\mu$, either $z_{1}$ or $z_{2}$ is always less than one. However, it is sometimes convenient to ignore this restriction to display the symmetries of the model. The complete partition function is given by

$$
Z=\int[d P] \prod_{x}\left[D_{x} \bar{D}_{x}\right] \prod_{\langle x y\rangle}\left[\sum_{R} c_{R}^{N_{t}} \chi_{R}\left(P_{x}\right) \chi_{R}\left(P_{y}^{\dagger}\right)\right]
$$

where the integral $[d P]$ is over Haar measure for the Polyakov loop $P_{x}$ on each spatial lattice site $x$ and the sum over $\langle x y\rangle$ is a sum over nearest-neighbor pairs.

\section{B. Symmetries}

The physics of $S U(N)$ static quarks at finite density is invariant under $\left(z_{1}, z_{2}\right) \rightarrow\left(z_{2}, z_{1}\right)$ provided we also switch our definition of particle and antiparticle operators. However, we need not switch operators at the special points where $z_{1}=z_{2}$. These are the points where $\mu=0$ and we have particle-antiparticle symmetry. Notice however that the identity

$$
\operatorname{det}\left[1+z_{1} P_{x}\right]=z_{1}^{N} \operatorname{det}\left[1+z_{1}^{-1} P_{x}^{\dagger}\right]
$$

also leads to an invariance and a symmetry. The factor of $z_{1}^{N}$ represents the Boltzmann factor for a completely filled state at a site. Although this factor of $z_{1}^{N}$ does contribute to the free energy, it does not affect expectation values. This operation reflects the equivalence between particles and holes: a particle (relative to the vacuum) is equivalent to $N-1$ holes (relative to the completely filled state) at the same site. In the special case where $z_{2}=0$, there is an exact particle-hole symmetry under $z_{1} \rightarrow 1 / z_{1}$. This extends to an approximate particle-hole symmetry when antiparticle effects are small. If we apply the above identity to antiparticles as well as particles, we obtain an exact symmetry of the complete theory: The model is invariant under the transformation $\left(z_{1}, z_{2}\right) \rightarrow\left(1 / z_{2}, 1 / z_{1}\right)$. Note however that this transformation takes the physical region $z_{1}, z_{2}<1$ into the unphysical region $z_{1}, z_{2}>1$.

Let us suppose we are in a low-temperature, large $\mu$ region where $z_{2} \ll 1$ and antiparticle effects can be neglected. Then the fermion determinant $D$ is real and there is no sign problem when $z_{1}=1$; this is precisely the absence of a sign problem at "half-filling" for static quarks. See [29] for an extensive treatment of this property. These symmetries are easily extended to any representation of the gauge group. Note that an alternative approach to including static quarks at finite density is to add a term

$$
\sum_{x}\left[z_{1} \operatorname{Tr}_{F} P_{x}+z_{2} \operatorname{Tr}_{F} P_{x}^{\dagger}\right]
$$

directly to the action. However, this is an approximation to lowest order in $z_{1}$ and $z_{2}$ of the effects of static fermions or bosons. It therefore misses the effects of Pauli blocking as well as the symmetries of the fermion determinant just discussed.

In addition to the invariances associated with $z_{1}$ and $z_{2}$, this model inherits from finite-density QCD invariance under the combined action of charge conjugation $\mathcal{C}$ and complex conjugation $\mathcal{K}$ [19, 20]. Charge conjugation takes $\operatorname{Tr}_{F} P_{x} \rightarrow \operatorname{Tr}_{F} P_{x}^{\dagger}$. It is a symmetry when $\mu=0$, but not when $\mu \neq 0$. Complex conjugation is an antilinear symmetry, 
changing not only fields but also complex-conjugating ordinary numbers. Like $\mathcal{C}, \mathcal{K}$ is a symmetry of the model only when $\mu=0$. $\mathcal{K}$ acts on $\operatorname{Tr}_{F} P$ to give $\operatorname{Tr}_{F} P^{*}=\operatorname{Tr}_{F} P^{\dagger}$. It is easy to see that the combined effect of $\mathcal{C} \mathcal{K}$ is to leave the action invariant. From this, it can be shown that all the eigenvalues of the transfer matrix are either real or are part of a complex conjugate pair. Due to the symmetry of the model, the transfer matrix $T_{s}$ commutes with $\mathcal{C} \mathcal{K}$. If $T_{s}|\lambda\rangle=\lambda|\lambda\rangle$ it follows that

$$
T_{s} \mathcal{C K}|\lambda\rangle=\mathcal{C K} T_{s}|\lambda\rangle=\mathcal{C} \mathcal{K} \lambda|\lambda\rangle=\lambda^{*} \mathcal{C K}|\lambda\rangle
$$

so $\lambda^{*}$ is an eigenvalue of $T_{s}$ if $\lambda$ is. Correlation functions of operators that couple to eigenstates of $T_{s}$ with complex eigenvalues will generally exhibit some amount of sinusoidally-modulated exponential decay rather than the usual exponential decay found in models with Hermitian actions [19, 20, 24, 25]. We will show below that this sinusoidal modulation is present in strong-coupling QCD with a finite density of static quarks.

\section{STRONG-COUPLING CALCULATION OF THE SPECTRUM}

In $1+1$ dimensions, the transfer matrix connecting one Polyakov loop $P_{x}$ to its nearest neighbor $P_{y}$ in a pure gauge theory can be written as

$$
T_{0}=\sum_{R} c_{R}^{N_{t}} \chi_{R}\left(P_{x}\right) \chi_{R}\left(P_{y}^{\dagger}\right)
$$

in the gauge-invariant basis where states are class functions of $P$ :

$$
\Psi(P)=\sum_{R} b_{R} \chi_{R}(P) .
$$

We can regard $T_{0}$ as acting on wave functions $\Psi(P)$ or alternately on an infinite vector of coefficients $b_{R}$. We refer to the latter representation as the group character basis. We are free to choose the lattice action as reflected by the coefficients $c_{R}$, provided they have the correct behavior in the continuum limit. Although the Wilson action is the most common lattice action, there is an infinite class of lattice actions that lead to the same continuum limit. Because we are interested in tracking the behavior of Polyakov loop correlation functions in many representations, we will need to keep the higher-order terms in the character expansion of the effective action. We will use the heat kernel action, for which the coefficients are

$$
c_{R}=\exp \left(-g^{2} C_{R} a^{2} / 2\right)
$$

where $C_{R}$ is the quadratic Casimir invariant for $R$. This has important advantages for us over the standard Wilson action. The expression for $c_{R}$ is simple and easy to calculate. In addition, it yields exactly the continuum results for string tensions for pure gauge theories in $1+1$ dimensions. See [27, 30, 31] for an explanation of other properties of the heat kernel action.

Using the identification $\beta=N_{t} a$, the transfer matrix $T_{0}$ has the form

$$
T_{0}=\sum_{R} \exp \left(-\beta g^{2} a C_{R} / 2\right) \chi_{R}\left(P_{x}\right) \chi_{R}\left(P_{y}^{\dagger}\right) .
$$

In the group character basis the Casimir operator is diagonal. For $S U(3)$, its eigenvalues are

$$
C(p, q)=\frac{(p+1)^{2}+(q+1)^{2}+(p+1)(q+1)}{3}-1
$$

where $(p, q)$ specify the particular irreducible representation of $R$ of the gauge group. Here $p$ represents the number of columns of one box and $q$ represents the number of columns of two boxes in Young tableau. The transfer matrix in the group character basis is

$$
T_{0}=e^{-\frac{g^{2} \beta a}{2} C\left(p_{1}, q_{1}\right)} \delta_{p_{1} p_{2}} \delta_{q_{1} q_{2}}
$$

In the pure gauge theory, the eigenvalues of $T_{0}$ determine the exponential decay of correlation functions:

$$
\left\langle\operatorname{Tr}_{R} P_{x} \operatorname{Tr}_{R} P_{y}^{\dagger}\right\rangle \sim \exp \left(-C\left(p_{1}, q_{1}\right) \frac{g^{2} \beta a}{2}|x-y|\right) .
$$


It is convenient to define the combination $g^{2} \beta / 2$ to be $m_{0}$, so that each representation $R$ is associated with a mass $m_{p, q} \equiv C(p, q) m_{0}$ in the pure gauge theory. The eigenvalues of the pure gauge theory transfer matrix $T_{0}$ are given by

$$
\lambda_{p, q}=e^{-\frac{g^{2} \beta a}{2} C\left(p_{1}, q_{1}\right)}
$$

so a mass $m_{p, q}$ can be extracted as

$$
m_{p, q}=-\frac{1}{a} \log \left(\frac{\lambda_{p, q}}{\lambda_{0,0}}\right)
$$

In the more general case where static quarks are present, the eigenvalues of the transfer matrix cannot be associated with a fixed group representation, and the corresponding eigenvectors in character space are linear combinations of group characters. In general, we simply number the eigenvalues sequentially starting at zero, and define the mass by

$$
m_{j}=-\frac{1}{a} \log \left(\left|\frac{\lambda_{j}}{\lambda_{0}}\right|\right)
$$

taking into account that the eigenvalues may be complex. Note that $m_{0}$ is simply a convenient mass scale, and not the mass of the ground state. If we consider $m_{j} / m_{0}$ in the limit where quark effects vanish, we obtain the Casimir operator $C(p, q)$. It is only in this sense that a given mass can be associated with a representation in the general case. In the case where $\lambda_{j}$ is complex, $\operatorname{Arg}\left(\lambda_{j}\right)$ determines the wavenumber for the oscillations. In general, we define

$$
\exp \left(-m_{j} a+i k_{j} a\right)=\frac{\lambda_{j}}{\lambda_{0}}
$$

so that $k_{j} a=\operatorname{Arg}\left(\lambda_{j} / \lambda_{0}\right)$ determines the period of oscillation for a given eigenvalue $j$. In all the cases considered here, the ground state is unique and $\lambda_{0}$ is real and positive, so $k_{j} a=\operatorname{Arg}\left(\lambda_{j}\right)$. We will take the lattice spacing $a$ to be 1 , and treat $m_{0}$ as the fundamental parameter of the pure gauge theory.

In the group character basis, $\operatorname{Tr}_{F} P$ and $\operatorname{Tr}_{F} P^{\dagger}$ act as raising and lowering operators and can be expressed in terms of Kronecker deltas

$$
\begin{aligned}
& \operatorname{Tr}_{F} P=\delta_{p_{1}, p_{2}} \delta_{q_{1}, q_{2}-1}+\delta_{p_{1}, p_{2}-1} \delta_{q_{1}, q_{2}+1}+\delta_{p_{1}, p_{2}+1} \delta_{q_{1}, q_{2}} \\
& \operatorname{Tr}_{F} P^{\dagger}=\delta_{p_{1}, p_{2}-1} \delta_{q_{1}, q_{2}}+\delta_{p_{1}, p_{2}+1} \delta_{q_{1}, q_{2}-1}+\delta_{p_{1}, p_{2}} \delta_{q_{1}, q_{2+1}}
\end{aligned}
$$

The effect of heavy static quarks can in turn be represented in the partition function by the fermion determinant

$$
D\left(z_{1}\right)=1+z_{1} \operatorname{Tr}_{F} P+z_{1}^{2} \operatorname{Tr}_{F} P^{\dagger}+z_{1}^{3}
$$

while the effect of antiquarks is represented by

$$
\bar{D}\left(z_{2}\right)=1+z_{2} \operatorname{Tr}_{F} P^{\dagger}+z_{2}^{2} \operatorname{Tr}_{F} P+z_{2}^{3}
$$

where $z_{1}$ and $z_{2}$ are the "activities" for quark and antiquark defined above. The overall transfer matrix including the effect of quarks and antiquarks can be written as

$$
T_{s}=T_{0}^{1 / 2} D\left(z_{1}\right) \bar{D}\left(z_{2}\right) T_{0}^{1 / 2} .
$$

This particular form is chosen so that $T_{s}$ is Hermitian when $z_{1}=z_{2}$. While the transfer matrix corresponding to pure gauge fields, $T_{0}$, is Hermitian, the final transfer matrix, $T_{s}$, that includes the effect of heavy quarks and antiquarks is no longer Hermitian. $\operatorname{Tr}_{F} P$ and $\operatorname{Tr}_{F} P^{\dagger}$ connects between different representations so they introduce off diagonal elements in the transfer matrix. Since $\operatorname{Tr}_{F} P$ and $\operatorname{Tr}_{F} P^{\dagger}$ are different for $\mu \neq 0$, the off-diagonal elements are no longer symmetric and the transfer matrix is non-Hermitian.

In $1+1$ dimensions, the results obtained from the transfer matrix are exact at any value of the coupling. In the strong coupling region, the results from $1+1$ dimensions are also valid in higher dimensions to leading order in the character expansion. This result was noted long ago by [32]. Consider the strong-coupling expansion for a free scalar field, as shown in Fig. 3. For on-axis correlation functions, say $\langle\phi(0) \phi(r \hat{x})\rangle$, the leading diagram is a line between the two points, the single path of minimal length. For off-axis correlation functions, there are multiple minimal-length paths in the taxicab metric, e.g. $|x|+|y|+|z|$ in $3+1$ dimensions, but this gives rise to a prefactor that does not 


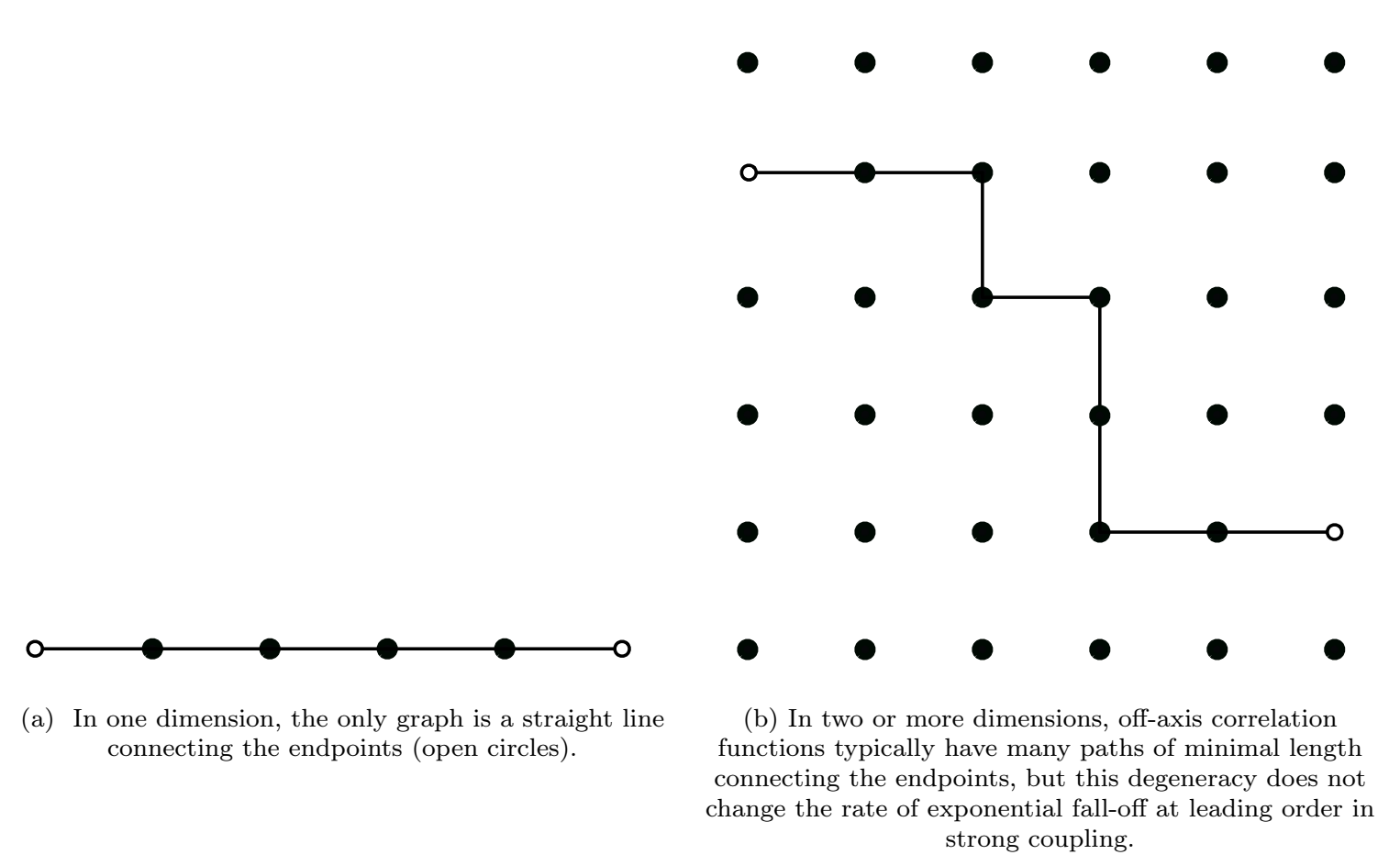

Figure 3: Strong-coupling diagrams for a free scalar theory.

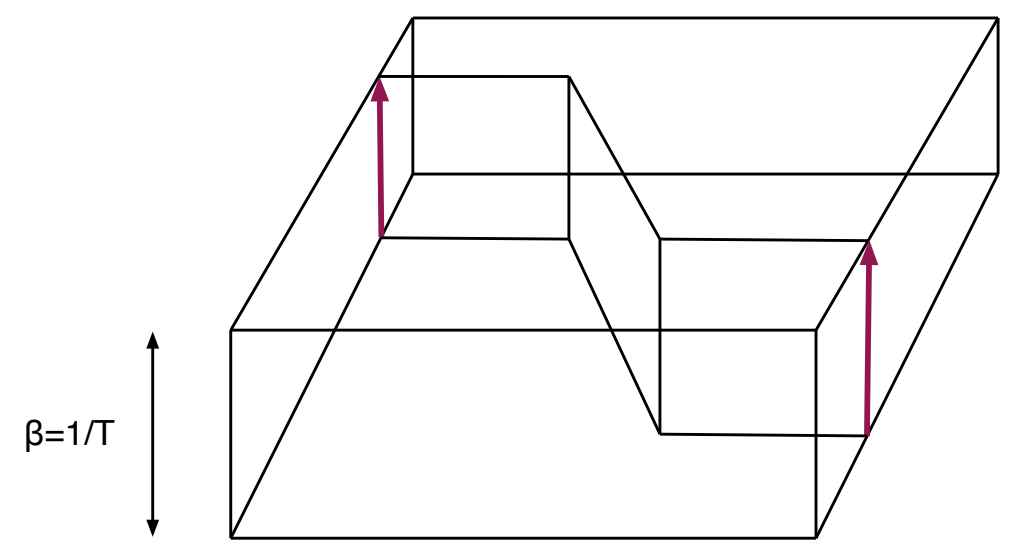

Figure 4: A contribution to the off-axis correlation function between two-widely separated Polyakov loops. For clarity, the intermediate staircase has been replaced by a diagonal sheet.

change the exponential decay of the correlation function. When looked upon as a spin system, the diagrammatic expansion here behaves similarly, so the leading-order strong-coupling result in $1+1$ dimensions is also the result in $d+1$ dimensions. Higher-order corrections to masses do explicitly depend on $d$. In any dimension, the strong-coupling expansion is a convergent expansion with a finite radius of convergence, so there will be some region around $g^{-2}=0$ where the lowest order result is a good approximation.

The analogous behavior for a gauge theory at finite temperature is shown in Fig. 4 below. For on-axis correlation functions of widely-separated Polyakov loops, the dominant contribution at strong coupling is a straight sheet, exactly as in $1+1$ dimensions. For the off-axis correlation function, there will be many minimal surfaces connecting the two Polyakov loops, but this will not change the rate of exponential fall-off at leading order in strong coupling. Thus we see that the strong-coupling results in $1+1$ dimensions are also valid in $d+1$ dimensions. 


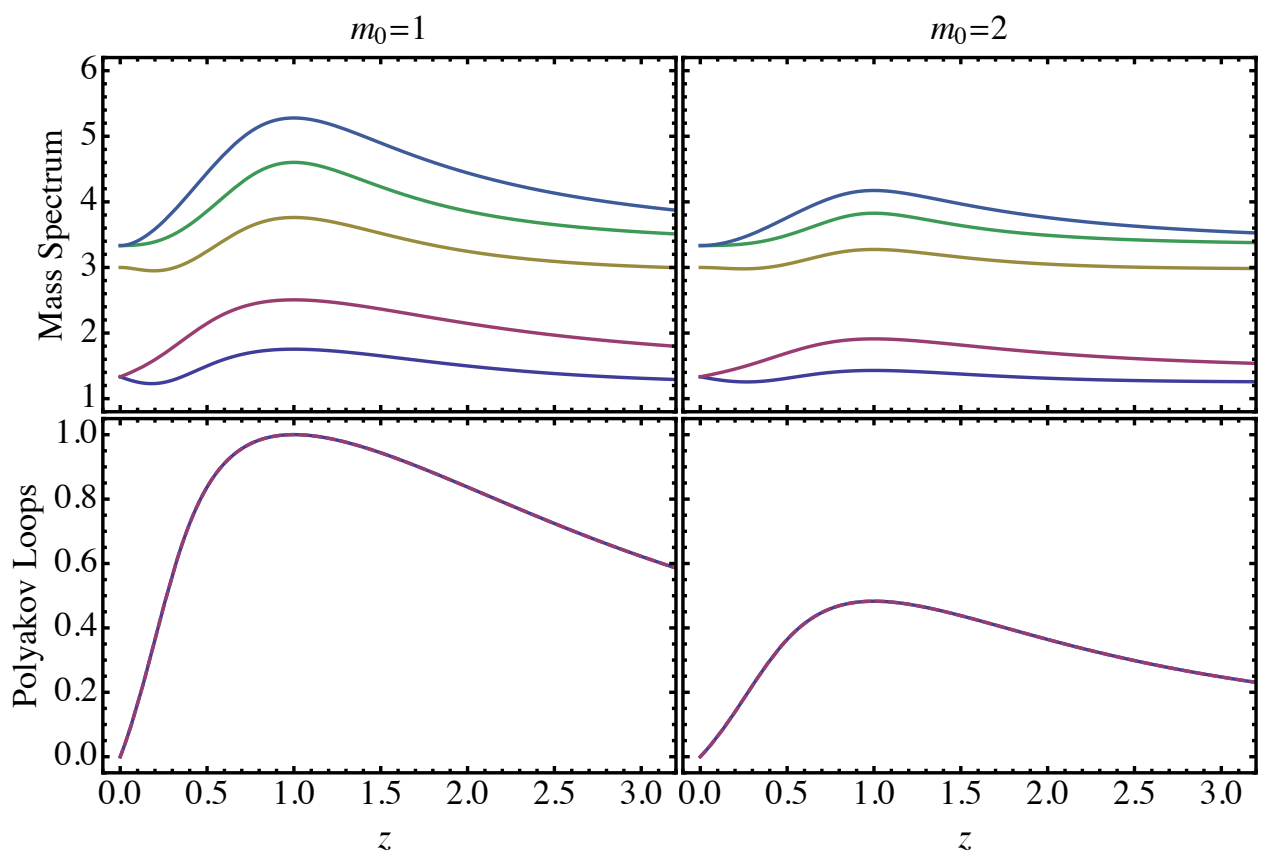

Figure 5: The mass spectrum and Polyakov loop expectation values for the $(1+1)$-dimensional $S U(3)$ model as a function of $z$ with $m_{0}=1$ and $m_{0}=2$.

\section{RESULTS FOR THE MASS SPECTRUM}

We now discuss our results for the low-lying eigenvalues of $T_{s}$. We begin with the case where $z_{1}$ and $z_{2}$ are set to a common value $z$, which corresponds to setting $\mu$ to zero so that $z$ can be identified with $\exp (-\beta M)$. In this case, the model is Hermitian so the eigenvalues are real and $\left\langle\operatorname{Tr}_{F} P\right\rangle=\left\langle\operatorname{Tr}_{F} P^{\dagger}\right\rangle$. Figure 5 shows the mass spectrum of the low-lying eigenstates and Polyakov loop expectations values for $m_{0}=1$ and 2 . The masses shown in the figures below are always divided by the mass scale $m_{0}$; the values of $m_{j} / m_{0}$ at $z=0$, on the left-hand axis, are thus the values of the Casimir operator for low-lying representations of $S U(3)$. In particular, we can associate the masses shown in Fig. 5 with the $3, \overline{3}, 8,6$ and $\overline{6}$ representations of $S U(3)$ when $z=0$. As can be seen in the figure, the values on the left-hand axis at $z=0$ are precisely $4 / 3,3$ and $10 / 3$. For $z>0$, the corresponding eigenvectors contain a mixture of the identity representation, the above five representations and other higher-dimensional representations. Both the spectrum and Polyakov loop expectation values are invariant under $z \rightarrow 1 / z$. This is reflected in the peaks achieved at $z=1$ by both the masses and the Polyakov loop expectation values. Physically, quark effects behave as an external magnetic field, and this effect is strongest at $M=0$, corresponding to $z=1$. Increasing $m_{0}$ from 1 to 2 shortens the correlation length in lattice units, and decreases the effects of the fermions on the spectrum. The peak in the Polyakov loop at $z=1$ is smaller at $m_{0}=2$ than at $m_{0}=1$ because the interaction between nearest-neighbor Polyakov loops is smaller. At $z=0$, pairs of complex representations like the 3 and $\overline{3}$ have degenerate masses. Because the quark determinant breaks the $Z(3)$ symmetry of the pure gauge theory, the eigenstates for $z>0$ do not show any degeneracy, but separate into clear, well-defined levels.

We next consider the case where the effects of antiquarks are neglected, which corresponds to setting $z_{2}=0$. Figure 6 shows the real and imaginary parts of the mass spectrum, $m_{j} / m_{0}$ and $\operatorname{Arg}\left[\lambda_{j}\right]$, for low-lying eigenstates when $m_{0}=1$ and 2. The figure also shows Polyakov loop expectation values. As seen in the plots, the masses start out real for $z_{1}=0$ but quickly take on complex values for non-zero $z_{1}$. As $z_{1}$ increases, the magnitude of complex part of the mass gradually increases before dropping back to zero. The plots clearly reflect the particle-hole symmetry under $z_{1} \rightarrow 1 / z_{1}$. The real part of mass spectrum is highest when $z_{1}=1$, which corresponds to $\mu=M$. The point $z_{1}=1$ is special because the theory is Hermitian at half-filling and the mass spectrum must be real. Furthermore, there appears to be a region around $z_{1}=1$ where the mass spectrum is real. The size of this region is largest for the 3 and $\overline{3}$ representations. The 8 representation does not develop an imaginary part. For $z_{1}<1,\left\langle\operatorname{Tr}_{F} P\right\rangle$ is less than $\left\langle\operatorname{Tr}_{F} P^{\dagger}\right\rangle$, implying that the free energy cost of inserting a fermion into the system is greater than that of inserting an antifermion. For $z_{1}>1$, this behavior is reversed in accordance with the $z_{1} \rightarrow 1 / z_{1}$ symmetry. At $z_{1}=1$, the two expectation values are equal. As in the case $z_{1}=z_{2}$, moving from $m_{0}=1$ to $m_{0}=2$ lessens the impact of fermions on the spectrum and on Polyakov loop expectation values. As mentioned earlier, this increase in scale of pure gauge 


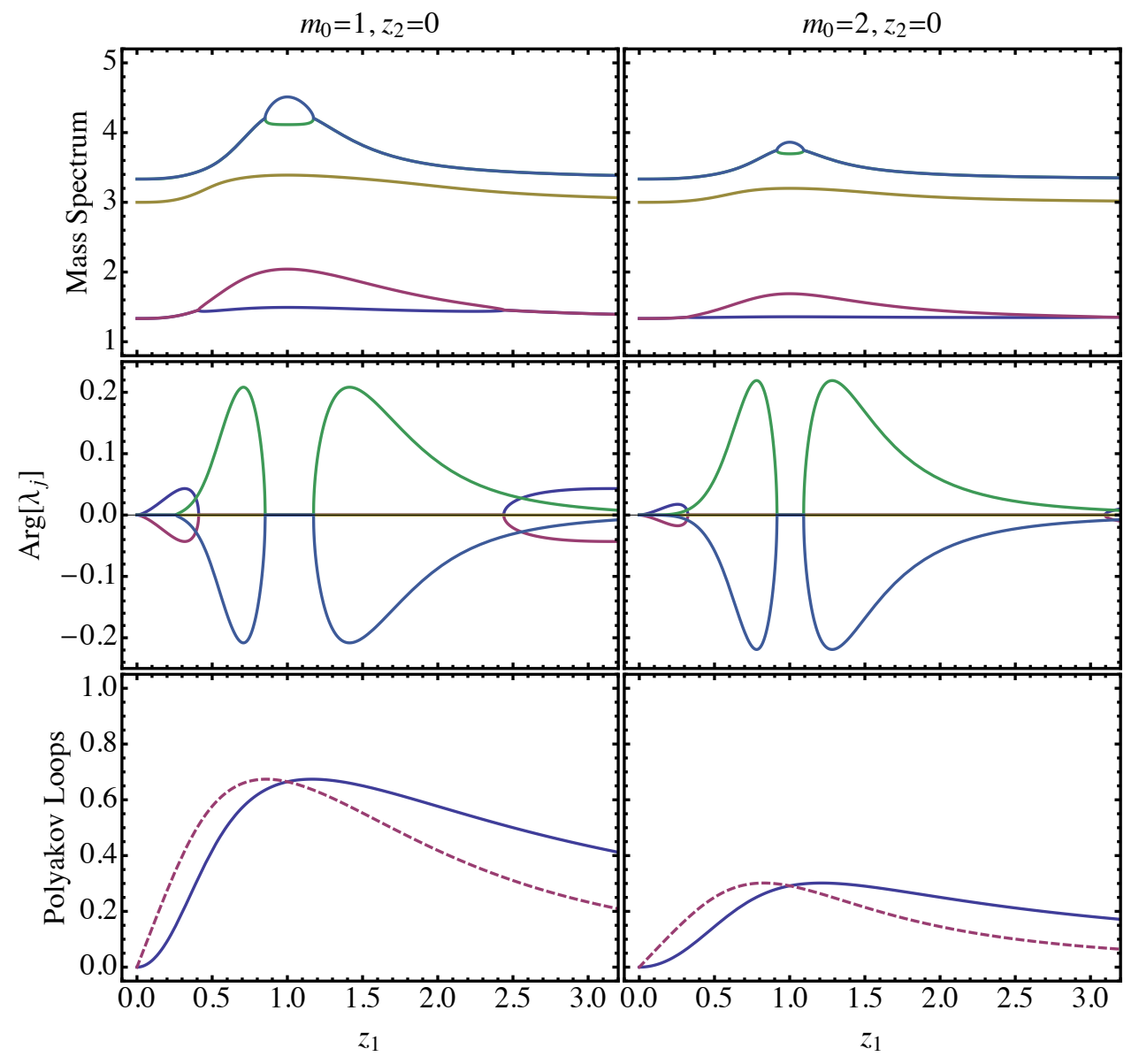

Figure 6: The real and imaginary parts of the mass spectrum and Polyakov loop expectation values for the $(1+1)$-dimensional $S U(3)$ model as a function of $z_{1}$ with $m_{0}=1$ and $m_{0}=2$ and $z_{2}=0$. The Polyakov loop $\left\langle\operatorname{Tr}_{F} P\right\rangle$ is represented by a solid line, and $\left\langle\operatorname{Tr}_{F} P^{\dagger}\right\rangle$ by a dashed line.

theory lessens the effects of fermions as seen in the plots.

We next consider the effects of antiquarks as gradually "turned on" by making $z_{2}$ non-zero. We fix the value of $m_{0}$ to 1. The region where $\operatorname{Arg}\left[\lambda_{j}\right]=0$ can be inferred from the non-degeneracy of the real parts. As seen from Fig. 7 . increasing the effect of antiparticles by making $z_{2}$ bigger gradually shrinks the region of complex mass. The region of complex mass are completely washed out for high enough value of $z_{2}$ and the mass spectrum is completely real. The value of $z_{2}$ for which the eigenvalues are real is smaller for low-lying eigenstates. For example, the 3 and $\overline{3}$ eigenstates are real even when $z_{2}$ is around 0.5 but we need $z_{2}$ to be around 1 for the eigenstates corresponding to 6 and $\overline{6}$ to be completely real. When $z_{2}$ is much larger than 1 , the mass spectrum will again show regions where $\operatorname{Arg}\left[\lambda_{j}\right] \neq 0$. This can be understood from the properties of the fermion determinants: the antifermion determinant at large $z_{2}$ is equivalent to a fermion determinant whose $z_{1}=1 / z_{2}$ is small. The spectrum at large $z_{2}$ is thus similar to that at small $z_{2}$.

We now turn to a more physical analysis of the spectrum in terms of the quark mass $M$ and the chemical potential $\mu$. In all cases, we set the fundamental scale-setting parameter $m_{0}=1$. The ratio $M / T$ is fixed at values between 0 to 5 and $\mu / T$ is varied from 0 to 6 . As shown in Figs. 8 and 9 , the behaviors of the mass spectrum and Polyakov loop expectation values are similar to what has been seen before, but the peak in the real part of the mass spectrum occurs near $\mu=M$, corresponding to $z_{1}=1$. As before, the eigenvalue associated with the 8 remains real throughout. When $M$ is large compared to $m_{0}$ and $\mu$, the spectrum is essentially that of the pure gauge theory. When $\mu$ is close to $M$ there is again a clear region where the low-lying eigenstates are real. In this region, there are well-defined eigenvalues associated with the 3 and $\overline{3}$, and with the 6 and $\overline{6}$. Near $\mu=M$, there are also clear maxima and minima in many of the mass values. This is presumably due to a relative maximum in the overall strength of $Z(3)$ breaking at that point. Furthermore, $\operatorname{Tr}_{F} P$ and $\operatorname{Tr}_{F} P^{\dagger}$ both peak near $\mu=M$, and also cross near this point. We attribute much of the observed behavior for $M \simeq \mu$ to an approximate particle-hole symmetry associated with the transformation $z_{1} \leftrightarrow 1 / z_{1}$. In the cases we are considering $z_{2}=\exp (-\beta \mu-\beta M)$ is typically less than 1 . This leads to an approximate 


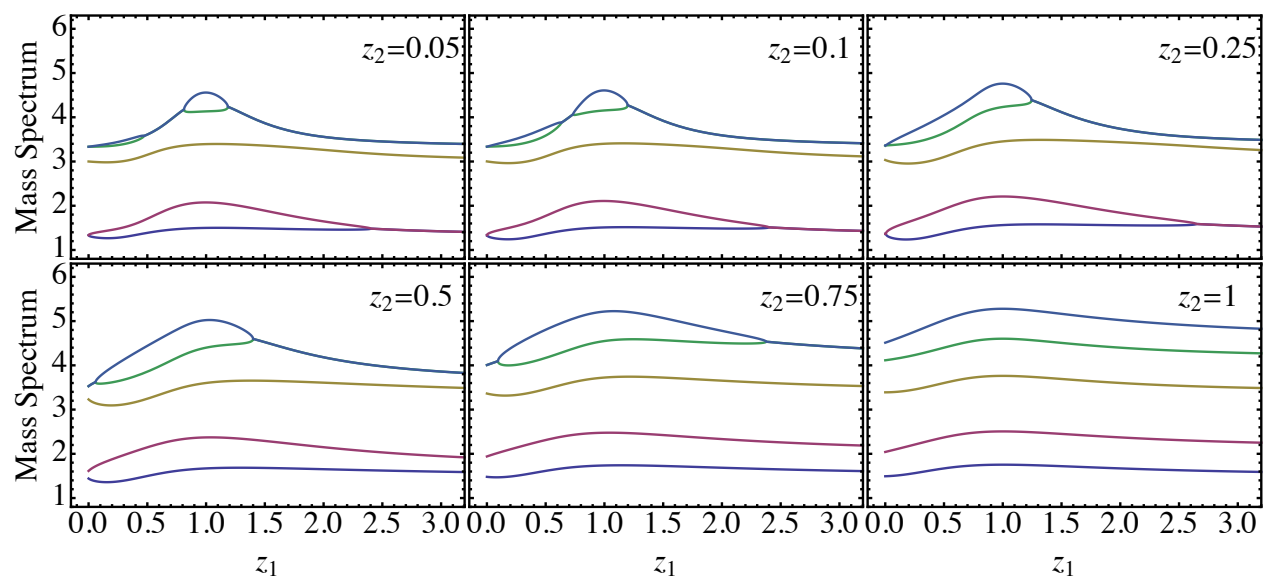

Figure 7: The real part of the mass spectrum of the $(1+1)$-dimensional $S U(3)$ model as a function of $z_{1}$ with $m_{0}=1$ and $z_{2}$ increasing.

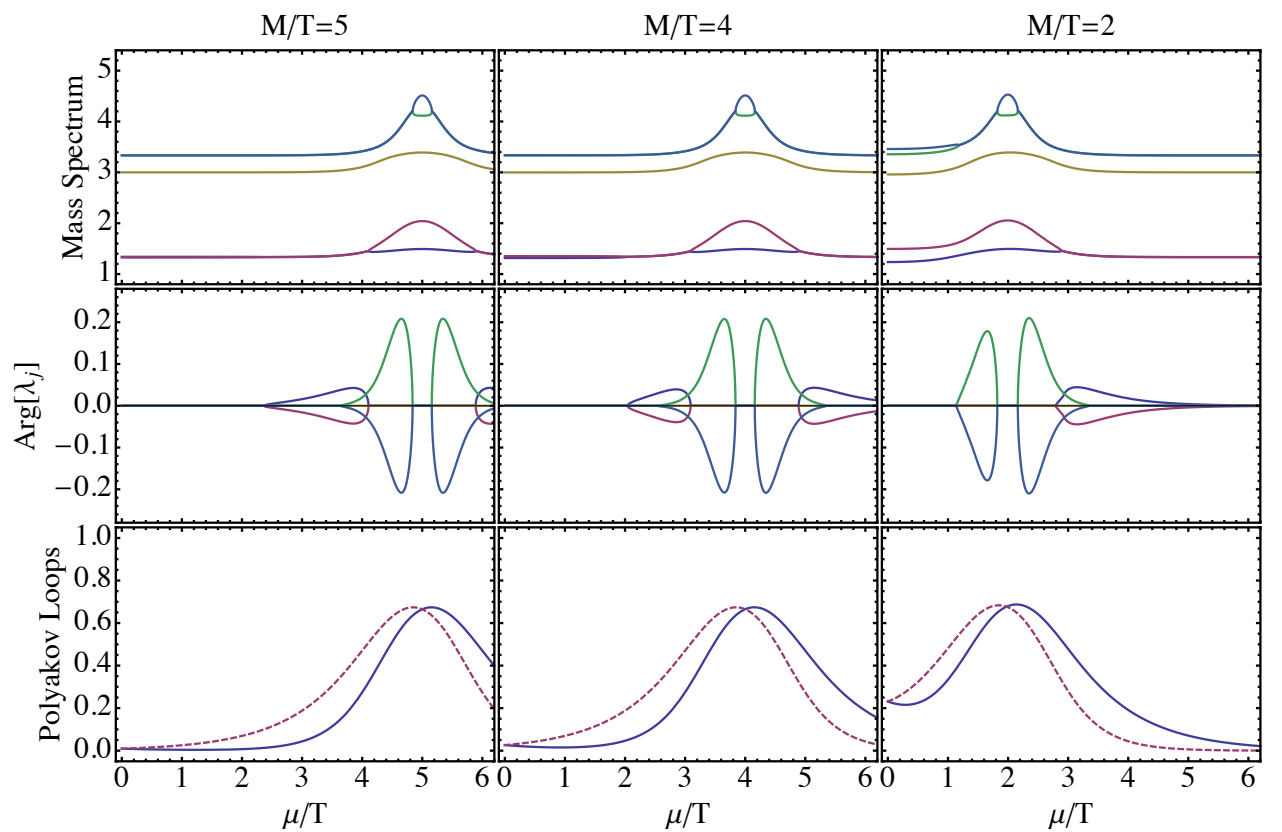

Figure 8: The real and imaginary parts of the mass spectrum and Polyakov loop expectation values for the $(1+1)$-dimensional $S U(3)$ model as a function of $\mu / T$ with $m_{0}=1$ and $M / T$ between 5 and 2 . The Polyakov loop $\left\langle\operatorname{Tr}_{F} P\right\rangle$ is represented by a solid line, and $\left\langle\operatorname{Tr}_{F} P^{\dagger}\right\rangle$ by a dashed line.

$z_{1} \leftrightarrow 1 / z_{1}$ symmetry with small corrections coming from $z_{2}<1$, with the effects of the symmetry most pronounced in the region $M \simeq \mu$. For $M / T$ large, $z_{2}$ is always much less than one, leading to an approximate particle-hole symmetry for all $\mu$; crossing of the Polyakov loops occurs at approximately the same value of $\mu / T$ as half-filling. For large $\mu$, Polyakov loop expectation values go to zero, also as a consequence of the approximate symmetry. Equivalently, one can view this as due to the saturation of the quark number density at large $\mu$. For $M / T \lesssim 0.55$, antiquark effects are significant and cannot be neglected: the low-lying spectrum is complex at half-filling.

On either side of the region including $M=\mu$, where the 3 and $\overline{3}$ are real, there are regions where the value of $\left|\operatorname{Arg}\left[\lambda_{j}\right]\right|$ for the 3 and $\overline{3}$ representation reaches a maximum. These regions also include the value of $\mu / T$ where $\operatorname{Tr}_{F} P$ and $\operatorname{Tr}_{F} P^{\dagger}$ are most different. A similar correlation of $\left|\operatorname{Arg}\left[\lambda_{j}\right]\right|$ with $\left|\left\langle\operatorname{Tr}_{F}\left(P-P^{\dagger}\right)\right\rangle\right|$ was seen in PNJL models [20]. As in Fig. 7, there is a second region where the low-lying eigenvalues are all real. This region is separate from the region around $M=\mu$ where the eigenvalues are real, and appears here for low $\mu / T$. This behavior is clearly visible for $M / T=2$, but is present for $M / T=4$ and even higher values.

As may be seen from Figs. 8 and 9 , the magnitude of the imaginary part of any eigenvalue is generally substantially 


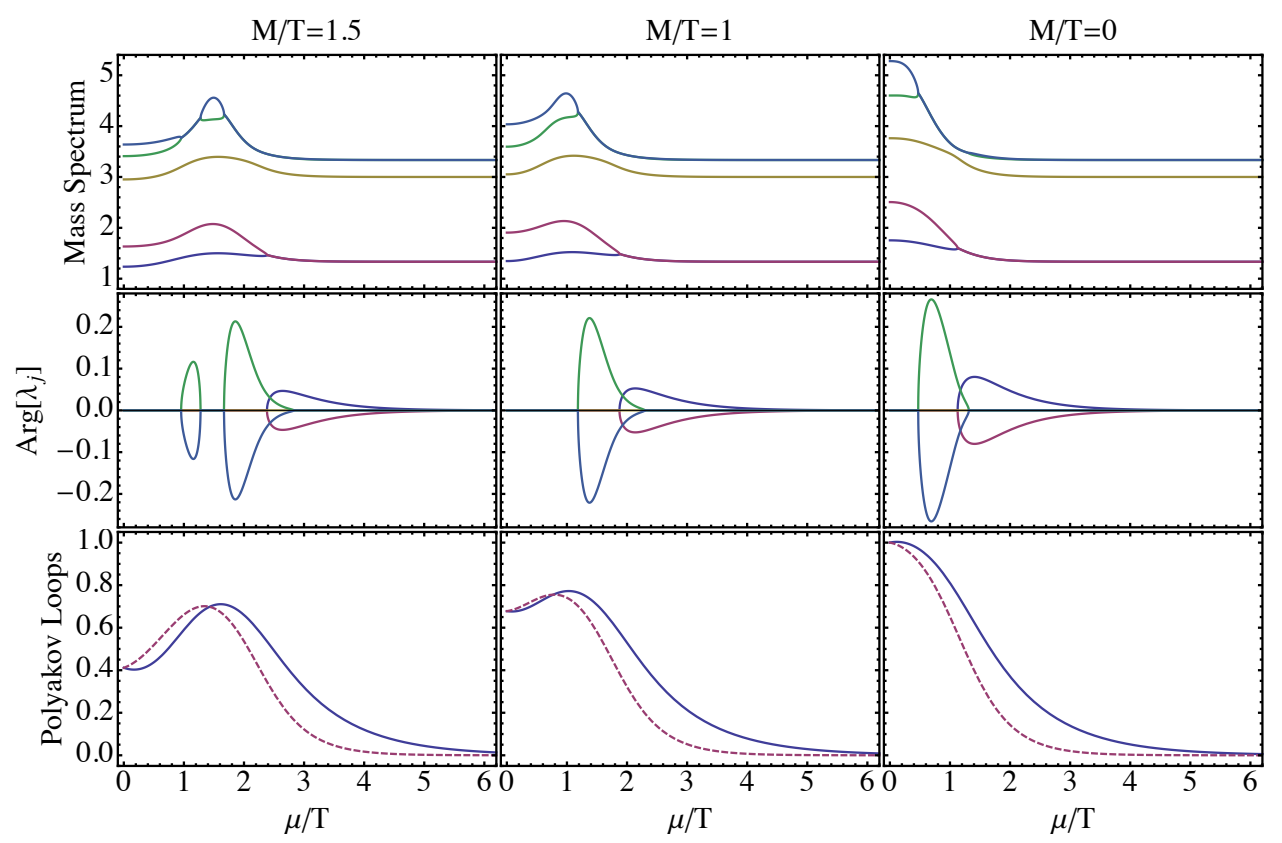

Figure 9: The real and imaginary parts of the mass spectrum and Polyakov loop expectation values for the $(1+1)$-dimensional $S U(3)$ model as a function of $\mu / T$ with $m_{0}=1$ and $M / T$ between 1.5 and 0 . The Polyakov loop $\left\langle\operatorname{Tr}_{F} P\right\rangle$ is represented by a solid line, and $\left\langle\operatorname{Tr}_{F} P^{\dagger}\right\rangle$ by a dashed line.

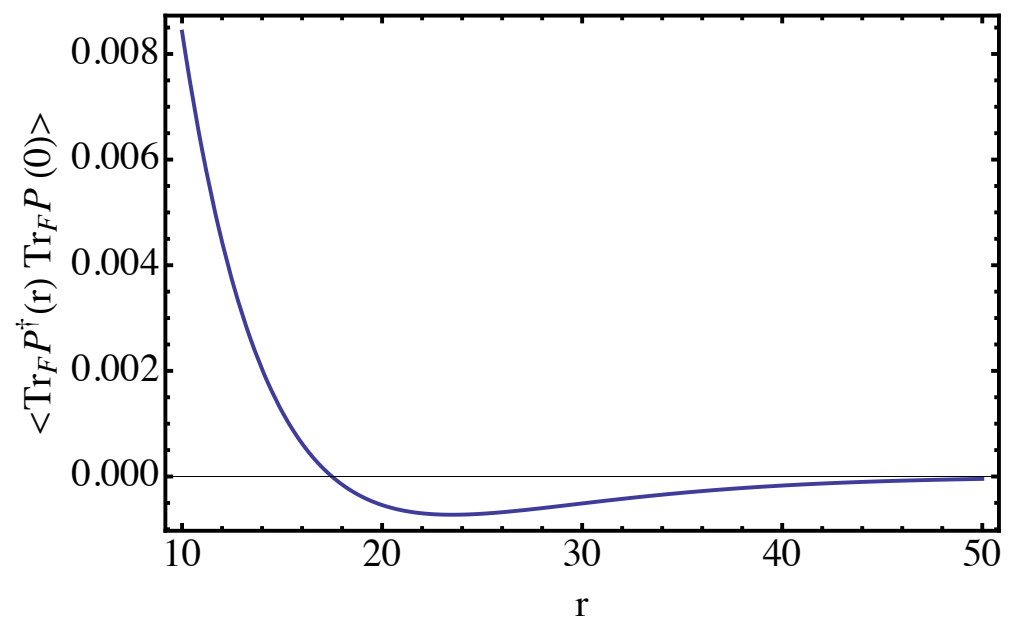

Figure 10: The $3-\overline{3}$ Polyakov loop correlation function $\left\langle\operatorname{Tr}_{F} P^{\dagger}(r) \operatorname{Tr}_{F} P(0)\right\rangle$ as a function of $r$ for $m_{0}=0.1$ and $z_{1}=0.08$ with $z_{2}=0$.

smaller than the real part and may be difficult to observe directly in simulations. Nevertheless, it may be possible to observe the modulated decay directly in some circumstances. Figure 10 shows the $3-\overline{3}$ Polyakov loop correlation function $\left\langle\operatorname{Tr}_{F} P^{\dagger}(r) \operatorname{Tr}_{F} P(0)\right\rangle$ as a function of $r$ for $m_{0}=0.1$ and $z_{1}=0.08$ with $z_{2}=0$. The clear minimum at $r \simeq 22$ is a consequence of sinusoidal modulation and reminiscent of the behavior of density-density correlation functions in liquids. Note also that the correlation function drops below zero, also as a consequence of the spatial modulation.

The quark number density also may be calculated. Results are shown in Fig. 11 for the quark number density as a function of $\mu / T$ for $M / T=1 / 2$ and $5 / 2$; the parameter $m_{0}$ is set to 1 . The most obvious feature is the saturation of the number density at 3 for large $\mu$. In the context of lattice gauge theories at finite density, saturation was first discussed in [33] for the case of $S U(2)$, where there is no sign problem. See [29, 34] for recent discussions of saturation effects in strong-coupling models of $S U(3)$ at finite density. Saturation is also observed in recent Langevin simulation with heavy quarks [8]. For the heavier quark mass $M / T=5 / 2$, we expect that antiquark effects are negligible for 


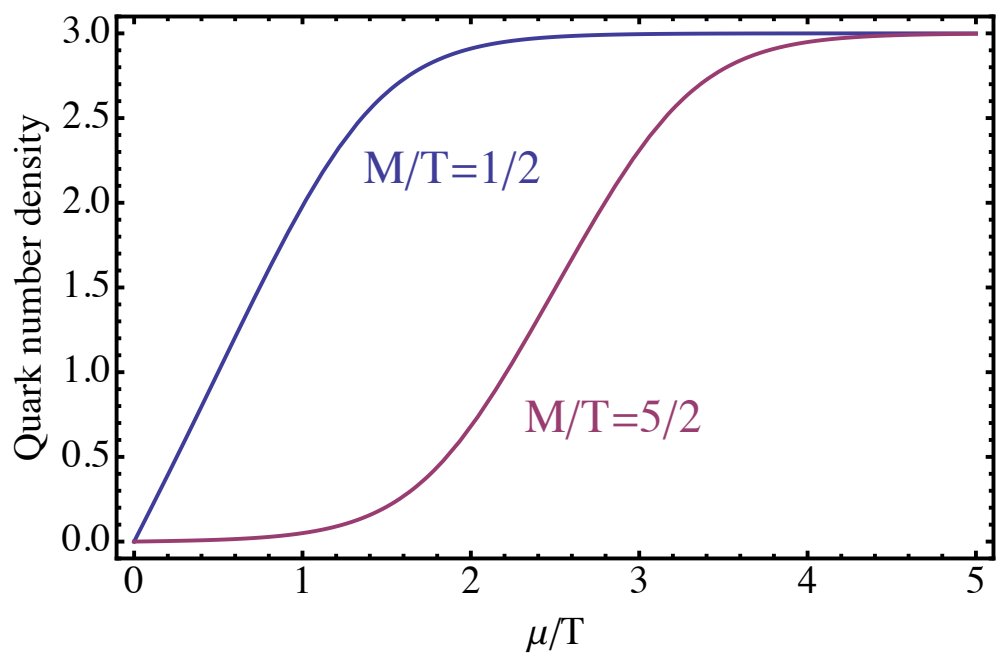

Figure 11: Quark number density as a function of $\mu / T$ for $M / T=1 / 2$ and $5 / 2$. The parameter $m_{0}$ is set to 1 . For the heavier mass $M / T=5 / 2$, the quark number density reaches half-filling (1.5) at $M=\mu$.

$\mu \simeq M$, and the system has an approximate particle-hole symmetry at $M=\mu$. This in turn implies that the quark number density reaches half-filling (1.5) at $M=\mu$. As may be seen from the figure, that expectation is confirmed. For the lighter quark mass, $M / T=1 / 2$, antiquark effects are not negligible when $\mu \simeq M$, and antiquark contributions lower the number density at $\mu=M$ below the half-filling value. In both cases, the number density saturates at some value of $\mu$ larger than $M$.

\section{CONCLUSIONS}

The spatial transfer matrix associated with Polyakov loops in finite-density QCD with static quarks have complex eigenvalues over a significant region of parameter space in strong-coupling limit. The appearance of complex eigenvalues is a direct consequence of the non-hermiticity of the transfer matrix. This is a manifestation of the sign problem in finite-density QCD. We have given a graphical explanation of the non-hermiticity in terms of the mixing between different representations. The invariance of finite-density QCD under $\mathcal{C K}$ symmetry ensures that the eigenvalues are either real or part of complex conjugate pair. The complex conjugate pairs in turn give rise to sinusoidal modulation of Polyakov loop correlation function. If the activities $z_{1}$ and $z_{2}$ are set to a common value $z$, i.e. $\mu=0$, all the eigenvalues are real. In this case, all the low-lying eigenvalues are largest when the static fermion mass $M$ is zero, corresponding to $z=1$. In the case where antiparticles effects are completely suppressed by setting $z_{2}=0$, the mass spectrum reflected the particle-hole symmetry of the theory under $z_{1} \rightarrow 1 / z_{1}$. As in the previous case, the real part of the transfer matrix eigenvalues peak at $z_{1}=1$, corresponding to $M=\mu$. This is a point where the transfer matrix is Hermitian, and also the point of half-filling. The complete suppression of antiquark effects obtained by setting $z_{2}=0$ is an approximation. If $z_{2}$ were exactly zero, then we would find that: a) all mass ratios would be real at $z_{1}=1$, a property that would hold in a region around $z_{1}=1 ; \mathrm{b}$ ) mass ratios would show maxima or minima at $z_{1}=1$; c) $\operatorname{Tr}_{F} P$ and $\operatorname{Tr}_{F} P^{\dagger}$ would cross at $z_{1}=1$. We have confirmed that these properties persist when $z_{2} \ll 1$, reflecting an approximate particle-hole symmetry. As $z_{2}$ increases and antiparticle effects become more important, the regions where complex eigenvalues occur reduce in size and eventually disappear as $z_{2}$ approaches $z_{1}$.

The mass spectrum can also be analyzed in terms of the more physical parameters $M / T$ and $\mu / T$. In general, the spectrum of low-lying eigenvalues shows a complicated set of behaviors, with both real and complex pairs of eigenvalues occurring. When $M / T$ is large compared to $m_{0}$ and $\mu / T$, the spectrum obtained is essentially that of pure gauge theory, and the low-lying eigenvalues are all real. The region where $\mu / T$ is close to $M / T$ corresponds to $z_{1}=1$, and the behavior in this region is largely determined by the approximate particle-hole symmetry discussed above. In all the cases studied, $\left\langle\operatorname{Tr}_{F} P\right\rangle \leq\left\langle\operatorname{Tr}_{F} P^{\dagger}\right\rangle$ for $0<\mu<M$ reflecting the lower free energy cost associated with introducing antiparticles. The occurrence of conjugate complex mass pairs and sinusoidal modulation of Polyakov loop correlation functions was previously observed by us in the study of phenomenological models of QCD using a saddle point approximations [19, 20]. The presence of similar phenomenon in lattice models of QCD at strong coupling strongly suggests the generality of the phenomenon. It has been suggested [35, 36] that sinusoidal modulation of this type might be observed in lattice simulations and heavy ion experiments. In both phenomenological models 
and in lattice strong-coupling calculations, the imaginary part of the mass is significantly smaller than the real part, suggesting that the direct observation of modulation might be difficult because of a long wavelength. However, there is another way to observe the splitting of the spectrum into complex pairs in lattice simulations. Suppose that the imaginary part of the masses are too small to be directly observed and can be neglected. In the regions where there are complex conjugate eigenvalue pairs, the real parts of the eigenvalues are degenerate, but outside of those regions they are different. This effect should be present and observable in lattice simulations of finite-density QCD, and may provide a strong test for finite-density algorithms.

\section{Acknowledgments}

HN thanks Olaf Kaczmarek, Sandra Klevansky, Jan Pawlowski, Dirk Rischke, Christian Schmidt and Ion Stamatescu for stimulating discussions. The research of HN was supported by Bielefeld University and GSI.

[1] Philippe de Forcrand. Simulating QCD at finite density. PoS, LAT2009:010, 2009.

[2] Sourendu Gupta. QCD at finite density. PoS, LATTICE2010:007, 2010.

[3] Gert Aarts. Complex Langevin dynamics and other approaches at finite chemical potential. PoS, LATTICE2012:017, 2012.

[4] Erhard Seiler, Denes Sexty, and Ion-Olimpiu Stamatescu. Gauge cooling in complex Langevin for QCD with heavy quarks. Phys. Lett., B723:213-216, 2013. doi: 10.1016/j.physletb.2013.04.062.

[5] Gert Aarts, Lorenzo Bongiovanni, Erhard Seiler, Denes Sexty, and Ion-Olimpiu Stamatescu. Controlling complex Langevin dynamics at finite density. Eur. Phys. J., A49:89, 2013. doi: 10.1140/epja/i2013-13089-4.

[6] Denes Sexty. Simulating full QCD at nonzero density using the complex Langevin equation. Phys. Lett., B729:108-111, 2014. doi: 10.1016/j.physletb.2014.01.019.

[7] Denes Sexty. Progress in complex Langevin simulations of full QCD at non-zero density. Nucl. Phys., A931:856-860, 2014. doi: 10.1016/j.nuclphysa.2014.09.029.

[8] Gert Aarts, Erhard Seiler, Denes Sexty, and Ion-Olimpiu Stamatescu. Simulating QCD at nonzero baryon density to all orders in the hopping parameter expansion. Phys. Rev., D90(11):114505, 2014. doi: 10.1103/PhysRevD.90.114505.

[9] Keitaro Nagata, Jun Nishimura, and Shinji Shimasaki. Justification of the complex Langevin method with the gauge cooling procedure. Progress of Theoretical and Experimental Physics, 2016(1), 2016. doi: 10.1093/ptep/ptv173.

[10] Marco Cristoforetti, Francesco Di Renzo, and Luigi Scorzato. New approach to the sign problem in quantum field theories: High density QCD on a Lefschetz thimble. Phys. Rev., D86:074506, 2012. doi: 10.1103/PhysRevD.86.074506.

[11] H. Fujii, D. Honda, M. Kato, Y. Kikukawa, S. Komatsu, et al. Hybrid Monte Carlo on Lefschetz thimbles - A study of the residual sign problem. JHEP, 1310:147, 2013. doi: 10.1007/JHEP10(2013)147.

[12] M. Cristoforetti, F. Di Renzo, A. Mukherjee, and L. Scorzato. Quantum field theories on the Lefschetz thimble. PoS, LATTICE2013:197, 2014.

[13] Marco Cristoforetti, Francesco Di Renzo, Abhishek Mukherjee, and Luigi Scorzato. Monte Carlo simulations on the Lefschetz thimble: Taming the sign problem. Phys.Rev., D88(5):051501, 2013. doi: 10.1103/PhysRevD.88.051501.

[14] Abhishek Mukherjee, Marco Cristoforetti, and Luigi Scorzato. Metropolis Monte Carlo integration on the Lefschetz thimble: Application to a one-plaquette model. Phys.Rev., D88(5):051502, 2013. doi: 10.1103/PhysRevD.88.051502.

[15] M. Cristoforetti, F. Di Renzo, G. Eruzzi, A. Mukherjee, C. Schmidt, et al. An efficient method to compute the residual phase on a Lefschetz thimble. Phys.Rev., D89:114505, 2014. doi: 10.1103/PhysRevD.89.114505.

[16] Gert Aarts, Lorenzo Bongiovanni, Erhard Seiler, and Denes Sexty. Some remarks on Lefschetz thimbles and complex Langevin dynamics. JHEP, 10:159, 2014. doi: 10.1007/JHEP10(2014)159.

[17] Yuya Tanizaki, Hiromichi Nishimura, and Kouji Kashiwa. Evading the sign problem in the mean-field approximation through Lefschetz-thimble path integral. Phys. Rev., D91(10):101701, 2015. doi: 10.1103/PhysRevD.91.101701.

[18] H. Fujii, S. Kamata, and Y. Kikukawa. Lefschetz thimble structure in one-dimensional lattice Thirring model at finite density. JHEP, 11:078, 2015. doi: 10.1007/JHEP11(2015)078.

[19] Hiromichi Nishimura, Michael C. Ogilvie, and Kamal Pangeni. Complex saddle points in QCD at finite temperature and density. Phys.Rev., D90:045039, 2014. doi: 10.1103/PhysRevD.90.045039.

[20] Hiromichi Nishimura, Michael C. Ogilvie, and Kamal Pangeni. Complex Saddle Points and Disorder Lines in QCD at finite temperature and density. Phys. Rev., D91(5):054004, 2015. doi: 10.1103/PhysRevD.91.054004.

[21] Kenji Fukushima. Chiral effective model with the Polyakov loop. Phys.Lett., B591:277-284, 2004. doi: 10.1016/j.physletb. 2004.04.027.

[22] Jean-Michel Drouffe and Jean-Bernard Zuber. Strong Coupling and Mean Field Methods in Lattice Gauge Theories. Phys. Rept., 102:1, 1983. doi: 10.1016/0370-1573(83)90034-0.

[23] Matthias Doring, Kay Huebner, Olaf Kaczmarek, and Frithjof Karsch. Color Screening and Quark-Quark Interactions in Finite Temperature QCD. Phys. Rev., D75:054504, 2007. doi: 10.1103/PhysRevD.75.054504.

[24] Michael C. Ogilvie and Peter N. Meisinger. PT Symmetry and QCD: Finite Temperature and Density. SIGMA, 5:047, 2009. doi: 10.3842/SIGMA.2009.047. 
[25] Michael Ogilvie, Peter Meisinger, and Timothy Wiser. $\mathcal{P} \mathcal{T}$ symmetry in statistical mechanics and the sign problem. International Journal of Theoretical Physics, 50:1042-1051, 2011.

[26] Benjamin Svetitsky and Laurence G. Yaffe. Critical Behavior at Finite Temperature Confinement Transitions. Nucl. Phys., B210:423, 1982. doi: 10.1016/0550-3213(82)90172-9.

[27] M. Billo, M. Caselle, A. D'Adda, and S. Panzeri. Finite temperature lattice QCD in the large N limit. Int. J. Mod. Phys., A12:1783-1846, 1997. doi: 10.1142/S0217751X97001158.

[28] Thomas C. Blum, James E. Hetrick, and Doug Toussaint. High density QCD with static quarks. Phys.Rev.Lett., 76: 1019-1022, 1996. doi: 10.1103/PhysRevLett.76.1019.

[29] Tobias Rindlisbacher and Philippe de Forcrand. Two-Flavor Lattice QCD with a Finite Density of Heavy Quarks: HeavyDense Limit and "Particle-Hole" Symmetry. 2015.

[30] M. S. Marinov and M. V. Terentev. DYNAMICS ON THE GROUP MANIFOLDS AND PATH INTEGRAL. Fortsch. Phys., 27:511, 1979. doi: 10.1002/prop.19790271102.

[31] P. Menotti and E. Onofri. The Action of SU(N) Lattice Gauge Theory in Terms of the Heat Kernel on the Group Manifold. Nucl. Phys., B190:288, 1981. doi: 10.1016/0550-3213(81)90560-5.

[32] J. B. Kogut, D. K. Sinclair, R. B. Pearson, John L. Richardson, and J. Shigemitsu. The Fluctuating String of Lattice Gauge Theory: The Heavy Quark Potential, the Restoration of Rotational Symmetry and Roughening. Phys. Rev., D23: 2945, 1981. doi: 10.1103/PhysRevD.23.2945.

[33] Simon Hands, Seyong Kim, and Jon-Ivar Skullerud. Deconfinement in dense 2-color QCD. Eur. Phys. J., C48:193, 2006. doi: $10.1140 /$ epjc/s2006-02621-8.

[34] Jens Langelage, Mathias Neuman, and Owe Philipsen. Heavy dense QCD and nuclear matter from an effective lattice theory. JHEP, 09:131, 2014. doi: 10.1007/JHEP09(2014)131.

[35] Apoorva Patel. Flux Tube Model Signals for Baryon Correlations in Heavy Ion Collisions. Phys.Rev., D85:114019, 2012. doi: 10.1103/PhysRevD.85.114019.

[36] Apoorva Patel. Baryon Number Correlations in Heavy Ion Collisions. PoS, LATTICE2012:096, 2012. 